\title{
Autonomous Object Manipulation Using a Soft Planar Grasping Manipulator
}

\author{
Robert K. Katzschmann, Andrew D. Marchese, and Daniela Rus
}

\begin{abstract}
This article presents the development of an autonomous motion planning algorithm for a soft planar grasping manipulator capable of grasp-and-place operations by encapsulation with uncertainty in the position and shape of the object. The end effector of the soft manipulator is fabricated in one piece without weakening seams using lost-wax casting instead of the commonly used multilayer lamination process. The soft manipulation system can grasp randomly positioned objects within its reachable envelope and move them to a desired location without human intervention. The autonomous planning system leverages the compliance and continuum bending of the soft grasping manipulator to achieve repeatable grasps in the presence of uncertainty. A suite of experiments is presented that demonstrates the system's capabilities.
\end{abstract}

\section{Introduction}

Q scale deformation, high compliance, and adjustable impedance compared to traditional rigid-bodied robots with high impedance. ${ }^{1}$ Such characteristics make this class of robots well-suited for highly dexterous tasks and interactions that require conformation to environmental uncertainty.

Our goal is to develop a soft planar fluid-powered gripper and a motion planning algorithm that leverages a soft morphology to robustly grasp, drag, and place objects of unknown geometry. In this article, we describe a planar soft robot manipulator we developed toward this goal. We focus on the design, fabrication, control, and planning aspects of this soft robot.

The fluid-powered gripper at the end of the arm can grasp an object through an open-loop controlled bending motion, even if the gripper is positioned relatively inaccurately in relation to the object to be grasped. The design of the gripper itself is inspired by the work of Polygerinos et al. ${ }^{2}$ The design is advantageous for grasping, because it exhibits high curvature, minimal radial expansion, and remains compliant during actuation. We can repeatably fabricate the gripper using a lost-wax casting process instead of the commonly used soft lithography technique. As a homogeneous piece without weakening seams, the gripper is not prone to delamination under high deformations. By abandoning the need for a lamination process, arbitrarily shaped internal channels can be achieved.

We attach the gripper to a multisegment soft manipulator to enable autonomous grasp-and-place capabilities on a plane. Positional feedback is provided in real-time from a camera system. Modeling uncertainties arise from a simplifying constant-curvature assumption, unrepresented manipulator dynamics, stick-slip friction, and nonlinear fluidic control. These uncertainties are compensated by the inherent compliance of our soft gripper design and our motion planning strategy. The motion planning algorithm advances the arm through all the necessary states of the grasp-and-place operation. A minimal strain, collision-free movement toward the object of interest is found by posing the plan as a series of constrained nonlinear optimization problems. The system first plans concentric approach circles shrinking from the initial end-effector pose down to the object diameter. Next, the system searches for locally optimal manipulator configurations that constrain the end-effector to lie on these approach circles, so that the manipulator does not collide with the object. The manipulator is then moved between these plans using closed-loop trajectories. After successfully approaching the object, the gripper encapsulates it. Even when the arm and gripper are fully actuated, they remain compliant, allowing it to conform to unmodeled object geometries. We experimentally validate the system's ability to repeatably and autonomously grasp and place randomly placed objects.

Computer Science and Artificial Intelligence Laboratory, Massachusetts Institute of Technology, Cambridge, Massachusetts.

(c) Robert K. Katzschmann et al. 2015; Published by Mary Ann Liebert, Inc. This Open Access article is distributed under the terms of the Creative Commons Attribution Noncommercial License (http://creativecommons.org/licenses/by-nc/4.0/) which permits any noncommercial use, distribution, and reproduction in any medium, provided the original author(s) and the source are credited. 


\section{Related work}

An overview of soft robotics is presented in Rus and Tolley. ${ }^{3}$ We will cover in the following the relevant works in fabrication, grippers, and control of soft robots.

Several manufacturing processes for soft biomimetic robots were reviewed by Cho et al. ${ }^{4}$ The vast majority of soft elastomer robots rely on the processes of soft lithography ${ }^{5}$ and/or shape deposition manufacturing. ${ }^{6}$ Especially noteworthy is the use of wax for fabricating jammable skin chambers, which stiffens by vacuuming. ${ }^{7}$ The gripper presented in this work also uses a lost-wax molding technique, but with a different type of actuation in mind: the obtained cavity structures are inflated to cause the gripper to bend.

There are several hardware examples for soft grippers described in recent literature; we will mainly focus on fluidicbased systems. Deimel and Brock developed a pneumatically actuated three-fingered hand made of reinforced silicone that is mounted to a hard robot and capable of grasping. ${ }^{8}$ More recently, they have developed an anthropomorphic soft pneumatic hand capable of dexterous grasps, which is not mounted to a robot but instead held by a human. ${ }^{9}$ Using a soft-lithography fabrication, Ilievski et al. created a pneumatic starfish-like gripper composed of an array of silicone chambers and a silicone membrane. ${ }^{10}$ The gripper hangs on a string and grasps objects like an egg or a mouse in an openloop controlled manner. Stokes et al. used a soft elastomer quadrupedal robot attached to a wheeled robot to grip and retrieve objects. ${ }^{11}$ A puncture-resistant soft pneumatic gripper was developed by Shepherd et al. ${ }^{12}$ An alternative to positive pressure actuated soft grippers is a robotic gripper that makes use of granular material jamming developed by Brown et al. ${ }^{13}$ Ikuta and Suzuki demonstrated a multiplesegment microhydraulic actuator for entering blood vessels. ${ }^{14}$ The soft octopus-inspired arms developed by Calisti et al. are not fluidic powered, but instead use cables to pull rigid fixtures embedded within an elastomer body. ${ }^{15,16}$ The arms were capable of grasping objects like pens or screws. A soft robotic tentacle developed by Martinez et al. was able to hold a flower and a horseshoe-shaped object. ${ }^{17}$ The closest related soft pneumatic actuator design to our current work is the fast Pneu-net designs by Mosadegh et al. ${ }^{18}$ and Polygerinos et al. ${ }^{2}$ These fingerlike actuators deform with minimal volume change and can bend to high curvatures. None of the above described grippers were controlled autonomously to perform their tasks, and accordingly no statement on repeatability of the autonomous execution was given.

Simulation results using an online motion planner for planar continuum manipulators were presented by Xiao and Vatcha. ${ }^{19}$ This work was extended by Li and Xiao to present a more general formulation to constrained, continuum manipulation. ${ }^{20}$ Marchese et al. demonstrated closed-loop position control of a multisegment soft planar fluidic elastomer manipulator in free space. ${ }^{21}$ The soft manipulator presented by Marchese et al. is only suitable for inspection tasks by moving through a constrained environment without object interaction or manipulation. $^{22}$

The work presented here combines two soft actuator types to leverage their strengths in a planar manipulation task. A new control method described here demonstrates the capabilities of this new soft manipulator to perform autonomous manipulations with uncertainties.

\section{Contributions}

We take on the challenge of grasping-and-placing objects with a seven degrees-of-freedom (DOF) planar arm made entirely from soft rubber. Our work differs from the previous work in that we create an entirely soft and autonomously controlled grasping manipulation system. Our planning and control method successfully copes with uncertainties in the object geometries, object placement, and manipulator modeling. We provide in this work the following contribution to soft robotics:

- The design and fabrication process of a soft 2D manipulator.

- A planning algorithm to grasp-and-place randomly positioned objects on a planar surface using a Seven DOF soft manipulator.

- Autonomous manipulation experiments with various objects of unknown geometry placed randomly in the working space of a soft manipulator without requiring force sensing or accurate positioning.

- Data from repeatable successful grasping demonstrations with a physical prototype and a qualitative experimental characterization of the uncertainty regions that can be tolerated by the soft gripper.

\section{System Overview}

The soft grasping manipulator shown in Figure 1 has six bidirectional segments with cylindrical cavities forming the arm and a single soft gripper with a pleated shape (Fig. 2) as the end effector. The independent pneumatic actuation of the unidirectional soft gripper and each bidirectional arm segment is achieved through an array of 13 custom fluidic drive cylinders. ${ }^{21}$ An object of feasible size but unknown geometry is randomly placed within the reachable envelop of the manipulator. The location of the manipulator and the object is determined with an external localization system. The motion planning algorithm as well as the curvature controller run on the control computers and take the location information as input. The curvature controller then provides continuous closed-loop adjustment of the fluidic drive cylinder array.

\section{Soft Grasping Manipulator}

The robotic manipulator is composed of multiple bidirectional planar arm segments and combined with a unidirectional soft gripper. We briefly describe the design, fabrication, and functionality of this soft grasping manipulator in this article. Further details on the design and fabrication can be found in Marchese et al. ${ }^{23}$

\section{Pleated channel design for the gripper}

The pleated channel design consists of evenly spaced ribs shown in cyan with embedded hollow sections shown in yellow. Cross-sectional views of the unactuated and actuated states are shown in Figure 2. This design approach draws inspiration for its pleats from the soft pneumatic gloves 


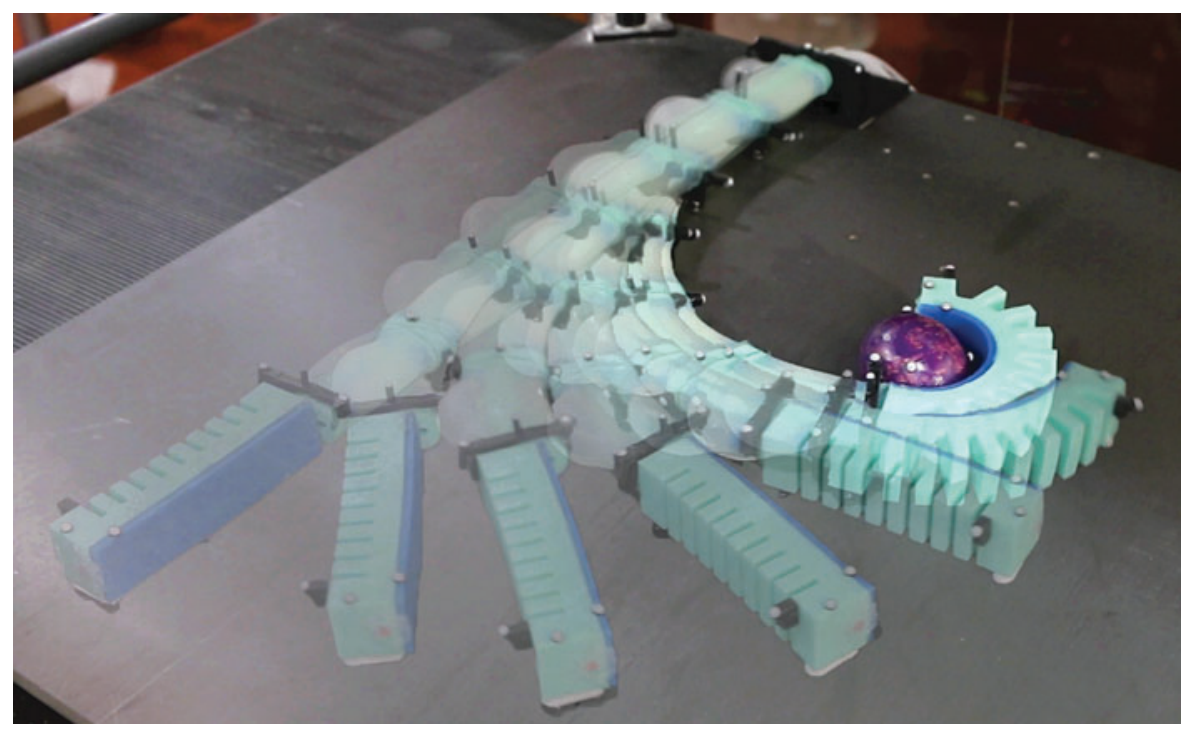

FIG. 1. The soft manipulator is grasping an egg. The robot repeatably approached, grasped, and moved this object.

developed by Polygerinos et al., ${ }^{2}$ and its homogeneous body design is inspired from the tail design of a soft robotic fish developed by Katzschmann et al. ${ }^{24}$ This design is advantageous for grasping because it exhibits high curvature, minimal radial expansion, and remains compliant during actuation. ${ }^{23}$ The hollow ribs within the segment's pleats are connected by a center channel and are accessible through a front inlet. Under fluidic pressurization of the interior channel, an individual pleat allows for a ballonlike expansion of the thin exterior skin along the axial direction. Similar to the uniform channel design, a stiffer silicone layer shown in blue serves as an almost inextensible constraint layer. The sum of the ballonlike expanding motions leads to the bending of the less extensible center constraint layer to form a grasp. The pleated design is capable of unidirectional bending up to extreme curvatures. Using a lost-wax casting approach, we are not limited in defining the geometry of the segment's fluidic channels. Using this approach, the cyan portion of the pleated gripper can be cured in a single step, avoiding any weakening seams due to lamination.

\section{Lost wax fabrication for fluidic elastomer actuators}

Existing soft fluidic manipulators are mostly produced through a multistep lamination process called soft lithogra- phy, which results in weakening seams that can easily delaminate. This limits their range of applications and lifetime. The retractable pin fabrication for uniform lateral channels, first introduced by Marchese et al., ${ }^{22}$ limits the complexity of the cavities to cylindrical shapes but does not cause weakening seams to the actuator. This is why the application of lost-wax casting to the fabrication of soft fluidic actuators like a gripper is advantageous. The actuated cavities of the soft gripper are achieved using a wax core, pourable silicone rubber, and 3D-printed molds. The gripper fabrication process and the tools needed are fully described and depicted in the section Grasp Object Planner and Figure 14 in Marchese et al. ${ }^{23}$

\section{Multisegment arm with gripper}

The design for the arm consisting of soft cylindrical segments is described in Marchese et al. ${ }^{22}$ As is shown in Marchese et al., ${ }^{23}$ through the characterization of various actuator morphologies, the concatenation of soft cylindrical segments is most suitable to build up a robotic arm that can create high blocking forces per fluid energy inserted. The cylindrical segments of the arm are fabricated through a retractable pin fabrication technique, ${ }^{22}$ which does not require lost wax cores because of their simple cylindrical cavities. Each cylindrical segment can be actuated up to a bend angle
A
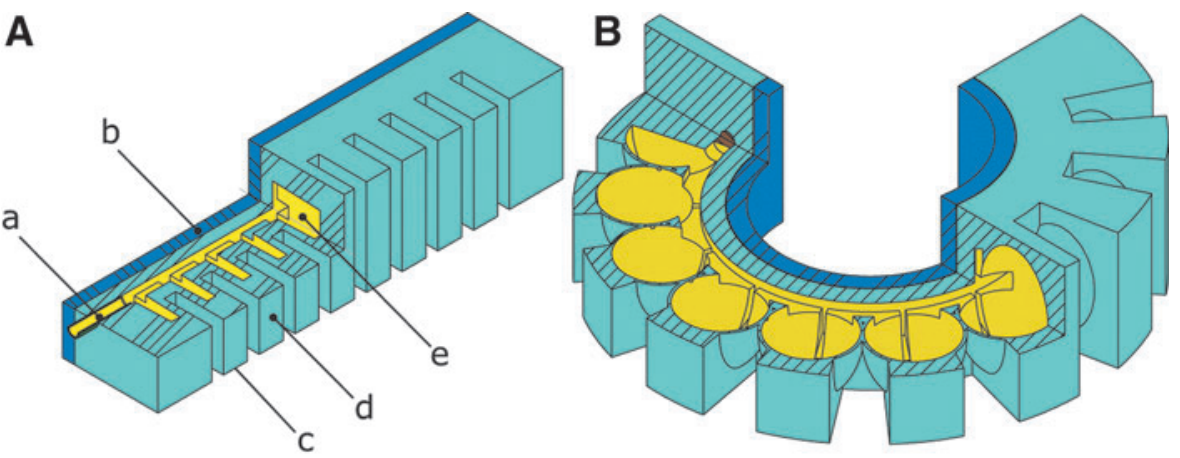

FIG. 2. The pleated channel designs. The design consists of a channel inlet (a), an almost inextensible constraint layer (b), uniform pleats (d) separated by even gaps (c), and internal channels within each pleat (e). (A) depicts the segment in an unactuated state and (B) shows the segment in an actuated and therefore bent state. The expansion of the pressurized channels are schematically represented. ( $\mathrm{Re}$ produced from Marchese et al. ${ }^{23}$ ) 
of about $60^{\circ}$; this requires several segments to be combined together to allow the arm to reach a large enough workspace to perform proper manipulation tasks on a plane. Using six segments, the robot is able to touch its tip to its base without interference from the individual joint limits. The cylindrical segment design with its hollow channel in the center has enough space to accommodate for pneumatic tubes to connect to all six cylindrical segments and additionally to the pleated gripper, which is attached to the tip. The pleated gripper has to be appropriately sized, just big enough to allow for proper manipulation without exceeding the payload capacity of the soft arm.

The complete multisegment arm is supported off the ground with two roller supports per segment. The rollers minimize frictional forces to the surface. If the arm would be moved over a nonslippery surface without rollers, the frictional effects would greatly reduce the agility of the arm and largely increase the stick-slip friction effects with the ground, rendering the arm less useful.

\section{Planning and Control}

This section covers our approach to preplanning motion waypoints for the soft robot and controlling the manipulator along those points.

\section{Kinematic control}

The forward kinematics algorithm forwKin ( ) assumes piecewise constant curvature. ${ }^{25}$ In order to uniquely fit a configuration representation to measured endpoint data in real-time, we use a previously developed single segment inverse kinematics algorithm singSegInvKin ( ). ${ }^{21}$ The inputs to this block are the start and endpoint measurements in $\mathbb{R}^{2}: \mathbf{E}_{n} \forall n=1 . . N$, where $N$ is the number of segments composing the arm. The outputs from this block are the represen- tations of the measured manipulator configuration: measured curvature $\boldsymbol{\kappa}_{\text {meas }}$ and segment length $\mathbf{L}_{\text {meas }}$. A cascaded closedloop curvature controller curvatureController () takes the target curvatures $\boldsymbol{\kappa}_{\text {target }}$ as its input and resolves the error between $\boldsymbol{\kappa}_{\text {target }}$ and $\boldsymbol{\kappa}_{\text {meas }}$ by continuously adjusting the fluidic drive cylinder array.

We build on the path planning algorithm presented in Marchese et al. ${ }^{22}$ This prior work plans the motion of a soft arm without a gripper through a maze at a centerline while taking the arm's bulging shape into account. The approach does not work for approaching and grasping objects, since a tip trajectory for successfully moving toward the object is not known, but needs to be generated by posing and solving a new optimization problem. The manipulator trunk should not push the object away when approaching it. Therefore, a new planner had to be developed and is presented in the following section.

\section{Autonomous grasp-and-place system}

The robotic manipulation system is capable of autonomously performing grasp-and-place operations. A state flow diagram describing its sensing, planning, and execution states is given in Figure 3. A motion tracker constantly captures the position of the object. The grasp object planner receives the coordinates and radius of the object and together with the current curvature values of the arm and gripper, it solves a series of constrained nonlinear optimization problems to generate end-effector poses approaching the object. Those end-effector poses are waypoints for an optimized path the robot arm should take to get to the final position without the risk of moving the object before the gripper grasps it. The intermediate waypoints ensure that the arm moves to the object while its null space maintains a convex shape, always bending away from the object. This is a conservative approach for not prematurely colliding with the object.
FIG. 3. State flow diagram of the planner developed for the autonomous grasp-and-place operation of the manipulator. This diagram describes essentially the flow of information from the motion tracking system to the discrete hardware.

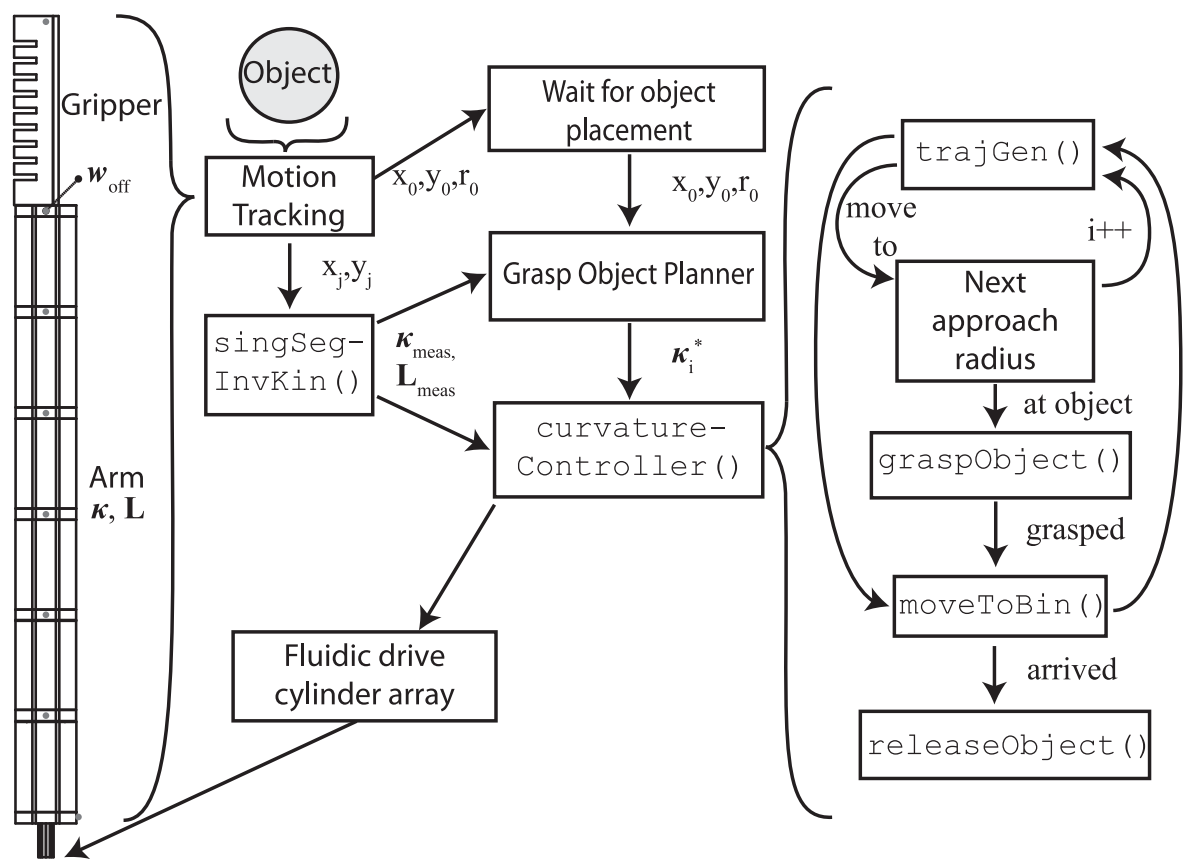


Furthermore, this approach allows the arm to move in smaller steps, decreasing the risk of large overshoots due to slip-stick friction between the roller supports and the ground. This planner is described in more detail in the section Grasp Object Planner.

The grasp object planner passes the approach configurations $\boldsymbol{\kappa}_{i}^{*}$ of the arm to the curvaturecontroller ( ) for execution in real-time. The controller receives measured curvatures $\boldsymbol{\kappa}_{\text {meas }}$ and lengths $\mathbf{L}_{\text {meas }}$ at an update rate of $100 \mathrm{~Hz}$ from the recursively called singSegInvKin ( ) and uses them to successively control the arm to every intermediate configuration $\boldsymbol{\kappa}_{i}^{*}$. During the arm initialization, the new curvature controller performs a prepressurization of both lateral channels. This is only done for the two segments closest to the root of the arm in order to stiffen them and shorten their response time constant. To allow for smoother transitions between each configuration $\boldsymbol{\kappa}_{i}^{*}$, we also added a trajectory generation procedure trajGen ( ) to the new curvature controller. It generates in real-time velocity profiles with acceleration and velocity constraints for each individual degree-of-freedom. These profiles allow real-time interpolation between the approach configurations of the arm while avoiding overshooting at the next target configuration. When the arm has arrived at the desired pose next to the object, the curvature controller initiates graspobject ( ). After encapsulating the object, moveToBin ( ) requests trajGen ( ) for another trajectory from the current pose to a predefined bin location. When the manipulator gets close to the bin location, the procedure releaseObject ( ) causes the gripper to open and release the object.

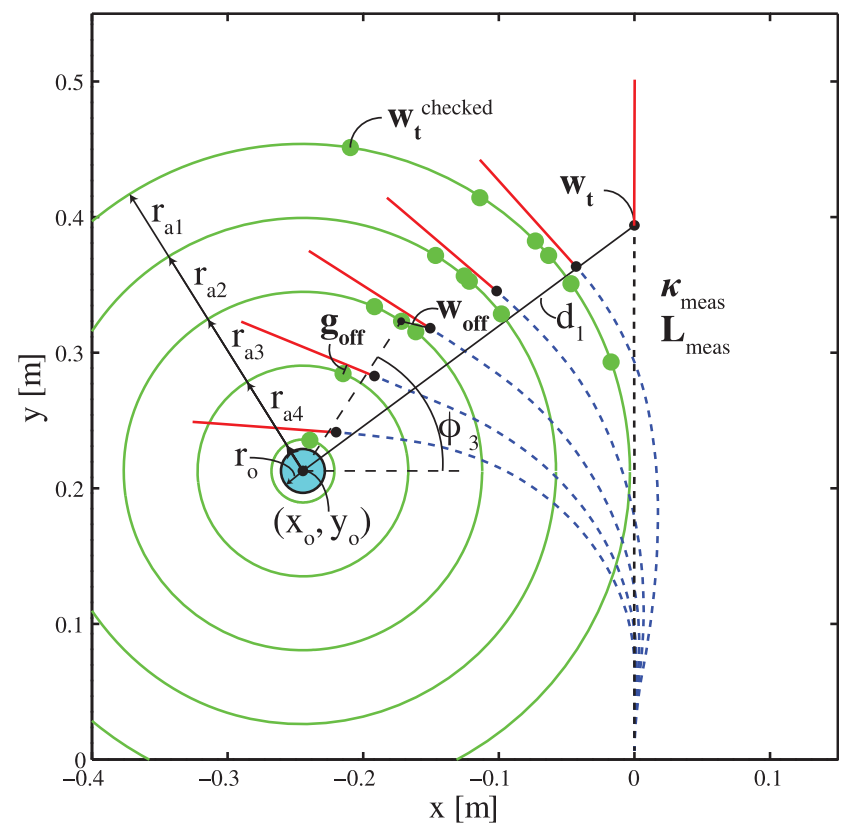

FIG. 4. Visualization of the grasp object planner. Concentric approach circles are centered about the object. The locally optimal approach configurations of the arm are shown as curved dashed lines with a straight gripper at the end. The initially measured manipulator configuration is shown as a long and dashed straight line on the right.

\section{Grasp object planner}

The grasp-and-place system plans a feasible approach motion to the object. That is, given the location $\left(x_{o}, y_{o}\right)$ and radius $r_{o}$ of a cylinder enclosing the object as well as the manipulator's current configuration $\boldsymbol{\kappa}_{\text {meas }}$ and segment lengths $\mathbf{L}_{\text {meas }}$, we determine a series of locally optimal manipulator configurations called approach configurations $\boldsymbol{\kappa}_{i}^{*} \forall i=1$.. numMoves that will, if sequentially achieved, bring the manipulator gradually closer to the object while any part of the arm is not touching the object.

The process for determining the approach configurations is detailed in the planGrasp ( ) procedure within the grasp object planner (see Algorithm 1). The planner is visualized in Figure 4. In short, we define a series of approach radii $r_{a i}$ $\forall i=1$..numMoves that define concentric circles shrinking from the manipulator's starting tip pose toward the center of the object. Given actuator limits, we then search for a series of feasible manipulator configurations $\boldsymbol{\kappa}_{i}^{*}$ that will place the robot's end-effector on the approach circles, parameterized by $r_{a}$ and $\phi$, while minimizing manipulator deformation. Minimized manipulator deformation is chosen as the optimization criterion, because it is proportional to the energy consumed by the fluidic drive cylinders, and it also minimizes the strain to the soft actuators.

The procedure planGrasp ( ) in Algorithm 1 first determines the manipulator's current tip pose $\mathbf{w}_{t}$ and the Euclidean distance $d_{1}$ between the tip and the object's center. The arc length input to the arm's forward kinematics is the $N$-th element of the segment lengths $\mathbf{L}_{\text {meas. }}$. The end effector offset $\mathbf{w}_{\text {off }}$ describes the distance from the root of the gripper to an offset point close to the lower end of the gripper's palm. It is visualized in the top left corner of Figure 3. The length $\mathbf{g}_{\text {off }}$ represents the component of the end effector offset $\mathbf{w}_{\text {off }}$, which is normal to the end effector orientation. The minimal tip transit distance $d_{2}$ is calculated by considering the object's radius $r_{o}$ and the gripper normal offset goff. Also, the number of approach configurations numMoves is determined as $\left\lfloor\frac{d_{2}}{\Delta d}\right\rfloor$, where $\Delta d$ is an allowable incremental distance. Using these parameters, approach radii $r_{a}$ shown by the green circles are iteratively calculated and their corresponding locally optimal configurations are found by using the optimization equation and constraints described in procedure findoptimalConfig $\left(r_{a i}\right)$ of Algorithm 1.

The procedure findoptimalConfig $\left(r_{a}\right)$ is posed as a nonlinear optimization problem. Here, the objective function represents the summation of independently weighted manipulator curvatures $\boldsymbol{\kappa}-\boldsymbol{\kappa}_{\text {off. }}$ The weights are set by the matrix $\mathbf{R}$. The variables to optimize for are $\phi$ and $\kappa$. The optimization constraints cause the manipulator's tip to lie on and be tangent to the approach circle. The constraints also ensure that the manipulator segment curvatures do not exceed the single soft actuator limits. Furthermore, this procedure leverages the arm's forward kinematics forwKin () defined in Marchese et al. ${ }^{21}$ and reproduced in the last section of Algorithm 1 for convenience. The optimization becomes over-constrained only if it has to find an arm pose outside of the arm's reachable workspace. That occurs if the object was user-placed outside the workspace. Before performing the optimization, a feasability check is performed using the arm's forward kinematics. 


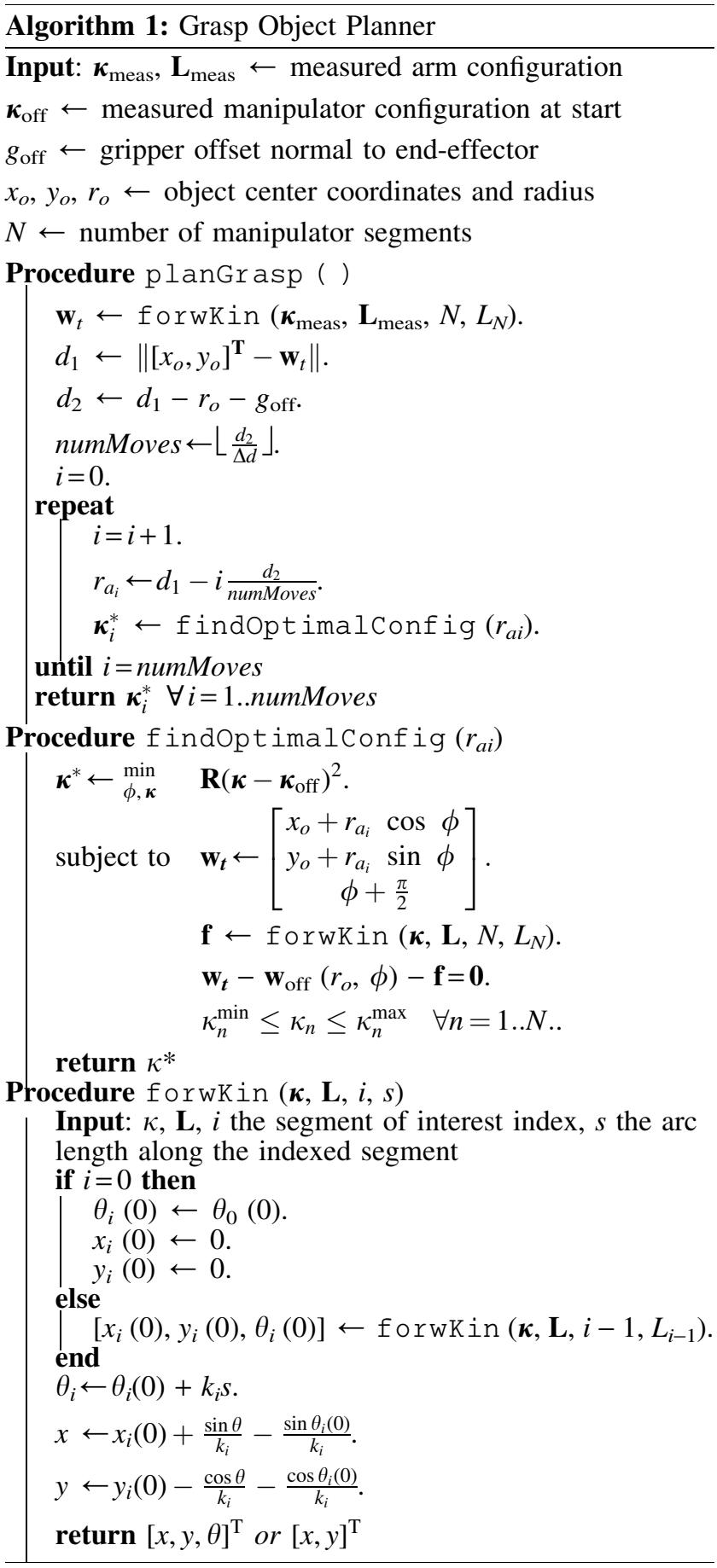

The nonlinear optimization problem is implemented on a PC using sequential quadratic programming, which finds iteratively the minimum of a constrained nonlinear multivariable function. The solver is run with a relative upper bound of $2 \times 10^{-3}$ on the magnitude of the constraint functions. The lower bound on the size of a step was given by $1 \times 10^{-6}$. The solver takes about $1 \mathrm{~s}$ to solve for all waypoints from start to finish.

\section{Experimental Results}

We now discuss the grasping of objects as well as the repeatability and success rate of the autonomous system.

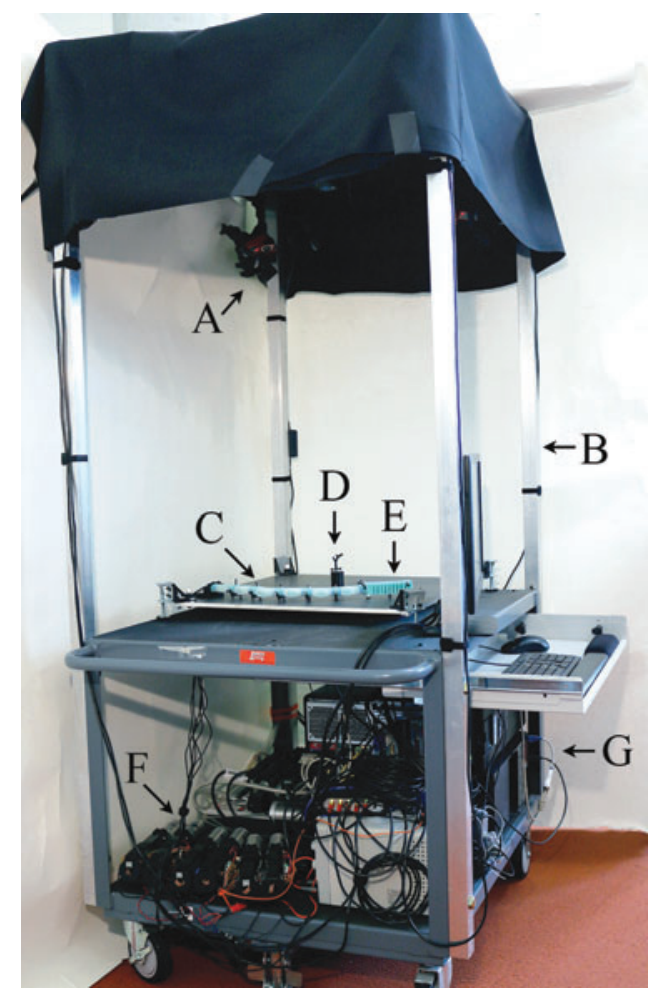

FIG. 5. System overview. The system is composed of (A) a motion capture system, (B) rigid frame, (C) soft six segment planar manipulator, (D) an object within the grasp envelope, (E) a soft gripper fixed to the manipulator, (F) a fluidic drive cylinder array to control actuation, and (G) computers for real-time processing and control.

\section{Experimental platform}

The soft manipulation system we developed for this work is shown in Figure 5. Each arm segment is $6.27 \mathrm{~cm}$, and the soft gripper is $10.6 \mathrm{~cm}$ long. The localization system OptiTrack Flex 3 by Natural Point provides real-time measurements of marked points both along the inextensible back of the manipulator and on top of the object. A rigid frame holds all the subsystems as a mobile presentation platform together providing reliable hardware experiments without the need for recalibration.

\section{Grasp experiments}

Using the experimental platform in Figure 5, we implemented the planning algorithm described in the Planning and Control Section. We evaluated the manipulator system for repeatability and ability to handle uncertainty. The experiments consisted of picking and placing several objects of unknown geometry at an unknown location. We measured the execution time and captured the location data during the experiments. Specifically, we performed over 200 experimental grasp-and-place trials at randomly chosen positions within the reachable workspace to demonstrate the capabilities and repeatability of our system. We successfully picked up various objects such as eggs, shuttlecocks, bakery items, cups, light bulbs, and tape holders. The objects had an enclosing diameter in the range of $2-5 \mathrm{~cm}$. The results of a subset of those experimental trials are shown in Figure 6. One representative approach, grasp, and retract move is shown in 

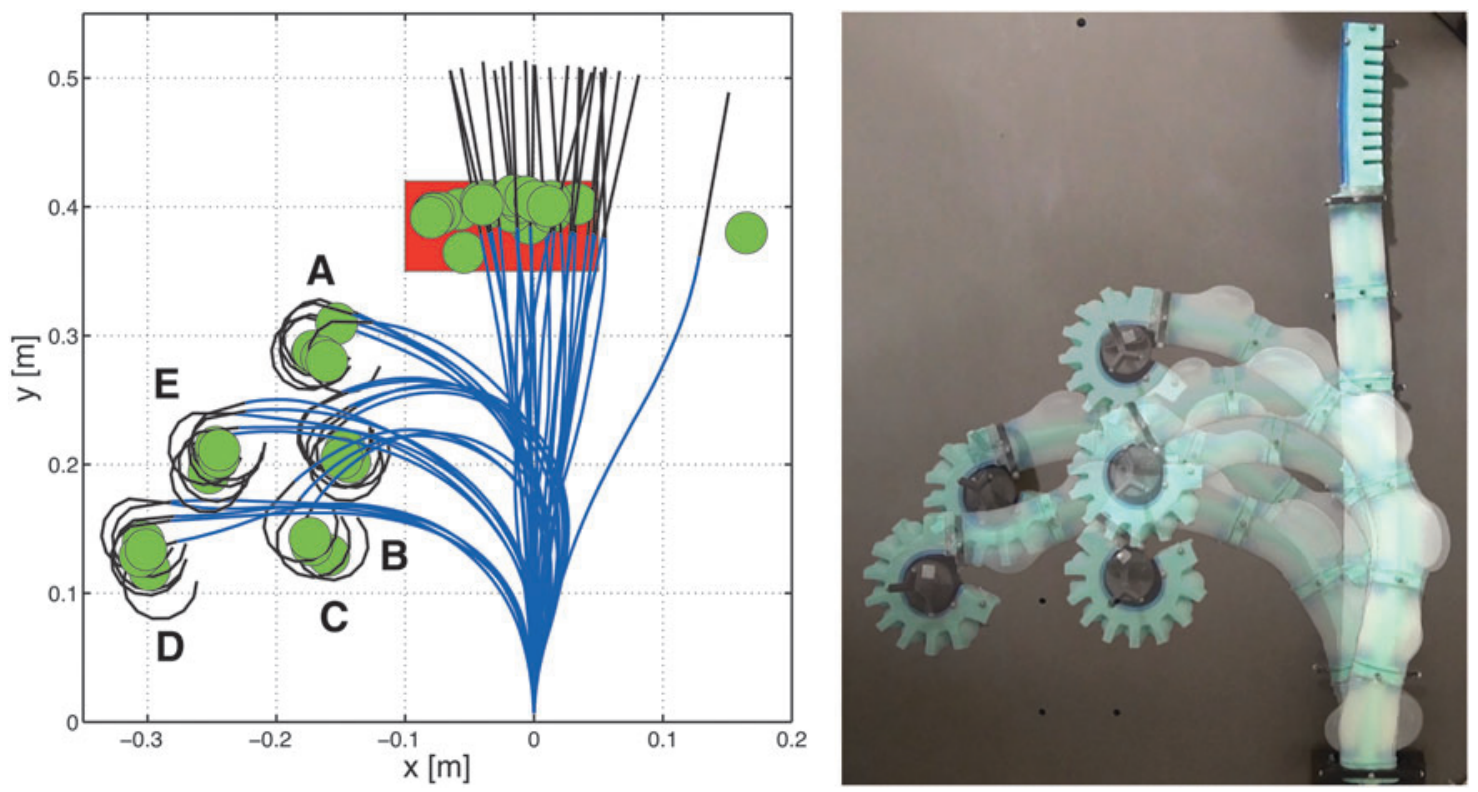

FIG. 6. (Left) Complete set of experimental grasp-and-place trials. In these experiments, the arm moves from an initial straightened configuration to grasp a round object placed in one of five locations (A-E). The arm then returns the object to a bin location shown in red. For each trial, a seven degrees-of-freedom (DOF) manipulator representation is generated at both the grasped and released state using experimental data and is shown in blue. The corresponding 1 DOF end-effector representation is shown in black. The round object's measured position at each state is shown in green. In one of the trials, the grasp and return was successfully performed, but an overshoot over the final bin location caused the gripper to drop off the small table it is moving on. (Right) Overlaid photographs of the manipulator grasping an object placed at each of the five locations.

Figure 7. In 23 of 25 experimental trials shown, the manipulator successfully achieved the task of grasping an object and placing it at a bin location shown in red. The test object has a weight of $18 \mathrm{~g}$ and a diameter of $3.3 \mathrm{~cm}$. The object was placed five times on each of the five points marked on the board. The markers only serve as a reference point for the user to place the object roughly at the same point at every repetition. The user's placing accuracy is not important to the algorithm, since the tracking system reregisters the position of the object every time it is placed. The five points were chosen to approximately represent the major portion of the manipulator's reachable workspace. As long as the root of the
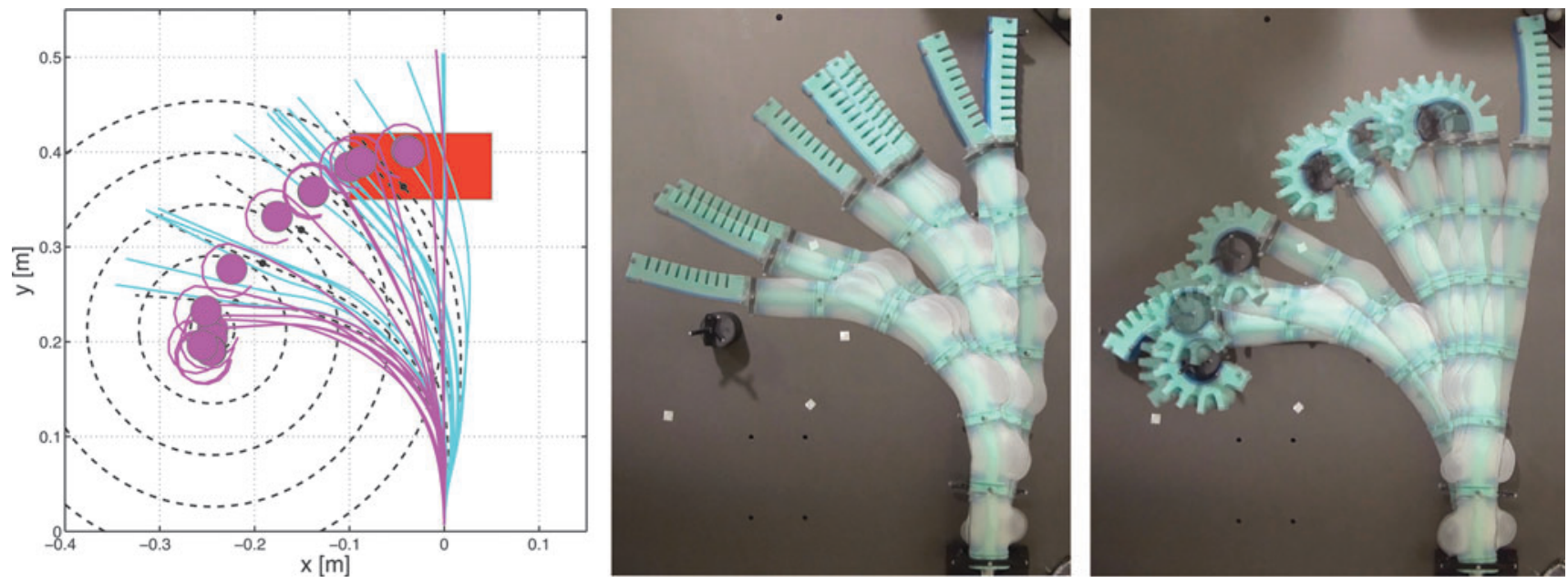

FIG. 7. (Left) A time series representation of an experimental grasp-and-place trial for an object located at point E (Fig. 6). Here, the locally optimal planned manipulator configurations as well as planned sequential approach circles are shown as black dotted curves. The arm and gripper are shown in their experimentally determined configuration representations at 1 intervals. The cyan configurations represent the manipulator prior to grasping the object that is moving from its initial configuration to the object's location. Depending on where the object is placed, the manipulator takes between 17-35s to approach it. After grasping the object, the magenta configurations represent the manipulator moving from the object's location back to the bin location shown in red. This task of moving back to the bin takes between 10-20s. (Right) Overlaid photographs of the manipulator moving from its initial pose to the object and from the object to the release location, respectively. 


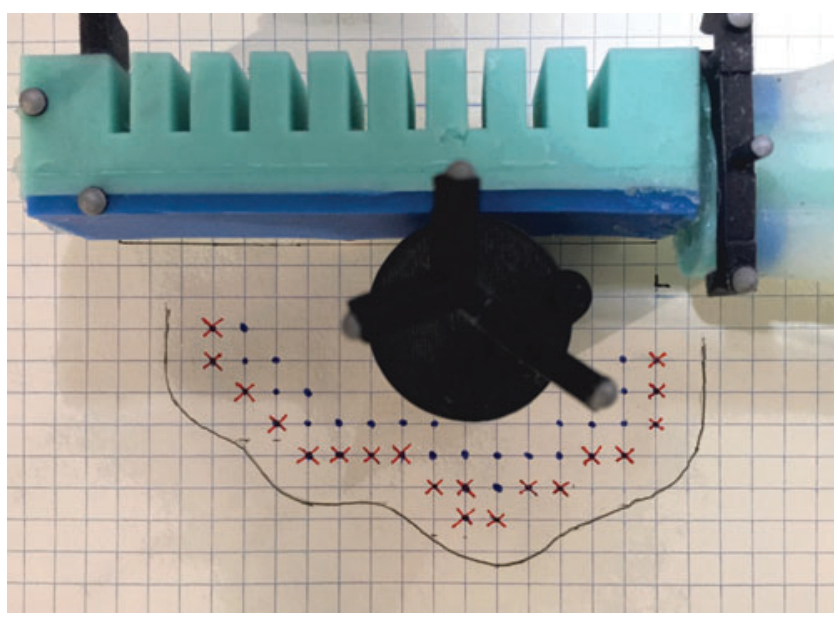

FIG. 8. Experimental characterization of the gripper's capture region: the allowable positioning uncertainty is determined through repeated placements of the center of a cylindrical object at different points on a $5 \mathrm{~mm}$, grid relative to the gripper. Blue dots indicate all object center positions for which a grasp could be performed successfully, red crosses show the positions where a grasp failed. The gray line outlines an area for the object to be positioned within so the gripper can grasp it. The evaluation of the capture region was performed similarly to a method described in Dogar and Srinivasa. ${ }^{26}$

gripper stops so that the object is located within the capture region, the gripper will pick it up through its sweeping closing motion. The capture region is outlined in gray in Figure 8.

The evaluation of the capture region is performed similarly to a method described in Dogar and Srinivasa's work on determining capture regions for a push-grasp of a classical robotic gripper. ${ }^{26}$ Grid paper and fine markings on all four sides of the round object ensure that the placement by the user is accurate within $\pm 1 \mathrm{~mm}$ in relation to the discrete placement locations on the grid. This test serves as a qualitative measure to show qualitatively a relation between object size to gripper size to area of successful grasp. This characterization was repeated two times, resulting in nearly identical capture regions. Despite positioning inaccuracies of the soft manipulator, the gripper can nevertheless successfully perform a grasp of an object. The successful capture region can be characterized by about half a gripper length in diameter.

When the arm closely approaches its straight pose, it drops the object. For these experiments, we focus on showing the capability of picking up objects at various places and moving them around; there is no emphasis set on having to drop off the object at a specific place. To indicate that the arm can move the object after grasping, the arm was controlled to go back to the fully straight pose. When the arm reached the final straight pose within a $1 \mathrm{~cm}$ delta, the gripper was set to release and drop the object. It was not ensured by the planner that the arm had to first settle to zero velocity at the final straight pose. As a consequence of this, the experimental data indicates as a red bin a relatively wide dropoff area.

The unsuccessful trials happened due to stick-slip friction between the roller bearings and the table surface. Our kinematic modeling does not account for this nonlinear behavior, which acts as a disturbance and can lead to failure to arrive at the next waypoint.

\section{Experimental insights and limitations}

Overall, the experiments show that the system was repeatably able to autonomously locate a randomly placed object within its workspace, plan the arm motions, and perform the task of grasping and placing the object. The system can drag payloads of less than $40 \mathrm{~g}$; higher payloads cause the cylindrical arm segments to stall and possibly lift off the table without moving the payload. There is a trade-off between the reachable workspace and the maximum payload. As the length of the arm increases, more workspace can be reached while less payload can be manipulated. A smoothing of the complete trajectory with several intermediate waypoints was found to be necessary. The amount of intermediate waypoints is determined by the variable $\Delta d$, which we found to be about the length of one arm segment.

We developed an end-to-end system that can approximately locate an object placed at an a priori unknown location and move it to a desired location. The external localization system is a convenient way to approximately identify the location of the object and to track how the object is moved around. The exteroceptive tracking system has the disadvantage that the full occlusion of one or more markers can cause the tracking system to temporarily lose track of a measured arm segment. In that case, the control loop can not function properly until the occlusion disappears. The external localization system could be replaced with another method for localizing the manipulator and the object in the workspace. For example, proprioceptive sensors within the segments could solve this issue partially. A first step toward proprioceptive sensing was done for three soft fingers arranged as a hand in Homberq et al. ${ }^{27}$

The experiments were performed for picking up objects on the left quadrant of the manipulator. Grasping objects on both sides of the manipulator could be achieved in various ways, including the following:

1. replacing the large gripper at the end of the arm with two smaller grippers next to each other,

2. mounting roller supports on the top face of the manipulator and then rotating the manipulator at its root by $180^{\circ}$, and

3. increasing the reachable workspace through starting the soft arm at an extreme curvature configuration within the right quadrant.

\section{Conclusion}

This work describes a planar soft manipulator capable of pick-and-place operations under high uncertainty in the position and shape of the object. A soft gripper was designed, fabricated, and combined with a previously developed soft robotic arm. It was then shown that a minimal strain and collision-free approach to an object of interest can be achieved by posing the grasp motion plan as a series of constrained nonlinear optimization problems.

The fabrication approach presented has potential to generalize beyond just the fabrication of a gripper. The new approach is advantageous because it allows for arbitrary designs of internal fluidic cavities and the casting of a homogeneous soft segment. It removes the need for laminating several separately casted parts together. Such a homogeneous soft segment would allow for better robot performance since it is less prone to manufacturing inconsistencies and rupture. 
The manipulator is suitable to perform delicate tasks with low payloads, for example, grasping objects that should not be squeezed and/or should not break during manipulation. The ability to successfully and repeatedly perform object manipulation using a fully soft, multiple degree-of-freedom arm suggests that despite their extreme compliance, soft robots are capable of reliable and robust object manipulation while simultaneously providing inherently safe interactions with the environment. We also demonstrated the manipulator's ability to autonomously grasp an object, which leads to many potential applications for full soft robotic manipulation. In a manufacturing setting, this could resemble a soft robot stretched widely to pick up objects situated at various locations. In a human-centric environment, soft arm grasping manipulation may enable soft robots to interact safely with humans. Future work will investigate the dexterity of the arm when approaching same object poses in various ways, just by changing the constraints and cost function when optimizing for the inverse kinematics solution. Integrating proprioceptive sensing within a multisegment soft actuator will further improve the use of these manipulators in occluded environments.

\section{Acknowledgments}

This research was conducted in the Distributed Robotics Laboratory at MIT with support from the National Science Foundation, grant numbers NSF 1117178, NSF IIS1226883, and NSF CCF1138967, and the National Science Foundation Graduate Research Fellowship Program, primary award number 1122374. We are grateful for this support.

\section{Author Disclosure Statement}

The authors declare that no competing financial interests exist.

\section{References}

1. Trivedi D, Rahn CD, Kier WM, Walker ID. Soft robotics: biological inspiration, state of the art, and future research. Appl Bionics Biomech 2008;5:99-117.

2. Polygerinos P, Lyne S, Wang Z, Nicolini LF, Mosadegh B, Whitesides GM, et al. Towards a soft pneumatic glove for hand rehabilitation. IEEE/RSJ International Conference on Intelligent Robots and Systems (IROS), IEEE, November 2013, pp. 1512-1517.

3. Rus D, Tolley MT. Design, fabrication and control of soft robots. Nature 2015;521:467-475.

4. Cho K-J, Koh J-S, Kim S, Chu W-S, Hong Y, Ahn S-H. Review of manufacturing processes for soft biomimetic robots. Int J Precis Eng Manuf 2009;10:171-181.

5. Xia Y, Whitesides GM. Soft lithography. Annu Rev Mater Sci 1998;28:153-184.

6. Cham JG, Bailey SA, Clark JE, Full RJ, Cutkosky MR. Fast and robust: hexapedal robots via shape deposition manufacturing. Int J Robot Res 2002;21:869882.

7. Steltz E, Mozeika A, Rodenberg N, Brown E, Jaeger HM. JSEL: jamming skin enabled locomotion. IEEE/RSJ Inter- national Conference on Intelligent Robots and Systems. (IROS), IEEE, October 2009, pp. 5672-5677.

8. Deimel R, Brock O. A compliant hand based on a novel pneumatic actuator. IEEE International Conference on Robotics and Automation (ICRA), IEEE, 2013, pp. 20472053.

9. Deimel R, Brock O. A novel type of compliant, underactuated robotic hand for dexterous grasping. Proceedings of Robotics: Science and Systems, Berkeley, July 2014.

10. Ilievski F, Mazzeo AD, Shepherd RF, Chen X, Whitesides GM. Soft robotics for chemists. Angew Chem 2011;123: 1930-1935.

11. Stokes AA, Shepherd RF, Morin SA, Ilievski F, Whitesides GM. A hybrid combining hard and soft robots. Soft Robot 2014;1:70-74.

12. Shepherd RF, Stokes AA, Nunes R, Whitesides GM. Soft machines that are resistant to puncture and that self seal. Adv Mater 2013;25:6709-6713.

13. Brown E, Rodenberg N, Amend J, Mozeika A, Steltz E, Zakin MR, et al. Universal robotic gripper based on the jamming of granular material. Proc Natl Acad Sci U S A 2010;107:18809-18814.

14. Ikuta K, Ichikawa H, Suzuki K. Safety-active catheter with multiple-segments driven by micro-hydraulic actuators. In: Dohi T, Kikinis R. (Eds). Medical Image Computing and Computer-Assisted Intervention MICCAI 2002 (Lecture Notes in Computer Science Series). Springer Berlin Heidelberg, 2002, pp. 182-191.

15. Calisti M, Arienti A, Giannaccini ME, Follador M, Giorelli M, Cianchetti M, et al. Study and fabrication of bioinspired octopus arm mockups tested on a multipurpose platform. 3rd IEEE RAS and EMBS International Conference on Biomedical Robotics and Biomechatronics (BioRob), IEEE, 2010, pp. 461-466.

16. Calisti M, Giorelli M, Levy G, Mazzolai B, Hochner B, Laschi C, et al. An octopus-bioinspired solution to movement and manipulation for soft robots. Bioinspir Biomim 2011;6:036002.

17. Martinez RV, Branch JL, Fish CR, Jin L, Shepherd RF, Nunes $\mathrm{R}$, et al. Robotic tentacles with three-dimensional mobility based on flexible elastomers. Adv Mater 2013;25: 205-212.

18. Mosadegh B, Polygerinos P, Keplinger C, Wennstedt S, Shepherd RF, Gupta U, et al. Pneumatic networks for soft robotics that actuate rapidly. Adv Funct Mater 2014;24: 2163-2170.

19. Xiao J, Vatcha R. Real-time adaptive motion planning for a continuum manipulator. IEEE/RSJ International Conference on Intelligent Robots and Systems (IROS), IEEE, 2010, pp. 5919-5926.

20. Li J, Xiao J. A general formulation and approach to constrained, continuum manipulation. Adv Robotics 2015;29: 889-899.

21. Marchese AD, Komorowski K, Onal CD, Rus D. Design and control of a soft and continuously deformable 2D robotic manipulation system. IEEE International Conference on Robotics and Automation (ICRA), IEEE, 2014.

22. Marchese AD, Katzschmann RK, Rus D. Whole arm planning for a soft and highly compliant 2D robotic manipulator. IEEE/RSJ International Conference on Intelligent Robots and Systems (IROS), IEEE, 2014. 
23. Marchese AD, Katzschmann RK, Rus D. A recipe for soft fluidic elastomer robots. Soft Robot 2015;2:7-25.

24. Katzschmann RK, Marchese AD, Rus D. Hydraulic autonomous soft robotic fish for 3D swimming. International Symposium on Experimental Robotics (ISER 2014), number 1122374, Marrakech, Morocco, 2014.

25. Webster RJ, Jones BA. Design and kinematic modeling of constant curvature continuum robots: a review. Int J Robot Res 2010;29:1661-1683.

26. Dogar MR, Srinivasa SS. Push-grasping with dexterous hands: mechanics and a method. IEEE/RSJ International Conference on Intelligent Robots and Systems (IROS), Oct. 2010, pp. 2123-2130.
27. Homberg B, Katzschmann RK, Dogar M, Rus D. Haptic identification of objects using a modular soft robotic gripper. IEEE/RSJ International Conference on Intelligent Robots and Systems (IROS), Sept. 2015.

Address correspondence to: Daniela Rus Computer Science and Artificial Intelligence Laboratory Massachusetts Institute of Technology 32 Vassar St. Combridge, MA 02139

E-mail: rkk@csail.mit.edu 